ESAIM: PROCEEDINGS, August 2010, Vol. 30, p. 153-165

D. Bresch, V. Calvez, E. Grenier, P. Vigneaux \& J.-F. Gerbeau, Editors

\title{
INFLUENCE OF THE SPRAY RETROACTION ON THE AIRFLOW*
}

\author{
L. Boudin ${ }^{1,2}$, C. Grandmont ${ }^{2}$, B. Grec $^{3,4}$ and D. Yakoubi ${ }^{2}$
}

\begin{abstract}
In this work, we investigate the influence of a spray evolving in the air, in the respiration framework. We consider two kinds of situations: a moving spray in a motionless fluid, and motionless particles in a Poiseuille flow. We observe that the spray retroaction may not be neglected in some situations which can really happen, for instance, when one considers rather big particles, as it is possible for polluting particles and even for some therapeutic aerosols. The retroaction is even responsible for increasing the deposition phenomenon.
\end{abstract}

\section{INTRODUCTION}

From therapeutic aerosols to polluting particles, a large scale of sprays, with respect to the particle densities, sizes or velocities, often interferes with our respiratory system. The spray inhalation may induce a mechanical effect during the breathing process. Indeed, the presence of particles in the airways can disturb the airflow itself. In this work, we study the influence of the spray on the airflow. The fluid has an indisputable effect on the spray through a drag (or friction) force. Conversely, one can very often find in the literature that the so-called retroaction of the spray on the air is neglected, see $[7,13]$ for instance. It is not neglected in [9], in the model as well as in the computations. This work aims to quantify this statement, as it was not investigated yet, up to our knowledge.

We do not tackle the question of the lung, airflow and spray models in this work. As in $[3,4,6,11,12,16,19,20]$, we assume that the airways have a tree structure, that the airflow obeys the incompressible Navier-Stokes equations down to the sixteenth generation of the respiratory tract, and that the spray is described by a Vlasov equation. The action of the spray appears as a source term in the Navier-Stokes equations. The relevance of the presence of this term is the main topic of this paper. Since the spray has first a local effect, we must point out that our study does not imply using biologically realistic geometries for the computations, so they are performed on a simple straight tube. Nevertheless, we also obtain global effects of the retroaction in our study, in particular on the spray deposition, which can be surprising at first glance.

The latter effect, i.e. the influence of the retroaction on the aerosol deposition, is one of the topics of an ongoing work in a more realistic three-dimensional setting, like a 3D branch. This work must be seen as the first step of the study of this retroaction term.

\footnotetext{
* This work was partially funded by the ANR-08-JCJC-013-01 project headed by C. Grandmont.

${ }^{1}$ UPMC Paris 06, UMR 7598 LJLL, Paris, F-75005, France;

e-mail: laurent.boudin@upmc.fr

${ }^{2}$ INRIA Paris-Rocquencourt, REO Project team, BP 105, F-78153 Le Chesnay Cedex, France;

e-mail: celine.grandmont@inria.fr \& driss.yakoubi@inria.fr

3 MAP5, CNRS UMR 8145, Université Paris Descartes, F-75006 Paris, France;

e-mail: berenice.grec@parisdescartes.fr

${ }^{4}$ Institut Camille Jordan, CNRS UMR 5208, Université Claude Bernard Lyon 1 / INSA Lyon, F-69622 Villeurbanne, France 
The paper is organized as follows. In the next section, we briefly describe our aerosol-air model originally presented in [4,6]. Then, in Sections 3 and 4, we study the influence of the aerosol on the air, in the case when the aerosol and air velocities do not have the same order of magnitude, i.e. when the spray is injected in a tube where the air is motionless, and when there are motionless particles in a Poiseuille profiled airflow. Eventually, in Section 5, we give some hints on the situations when one must take the retroaction into account in the model.

\section{Aerosol MODELling AND Simulation}

\subsection{Model}

The situations we deal with in this work are only two-dimensional, but the model also holds for three dimensions $[4,6,9,17]$. The airflow can be described by its velocity field $u(t, x) \in \mathbb{R}^{2}$ and the pressure $p(t, x) \in \mathbb{R}$, where $t \geq 0$ is the time and $x=\left(x_{1}, x_{2}\right) \in \mathbb{R}^{2}$ is the space location. Since the air is assumed to be incompressible, the air density remains constant, denoted by $\varrho_{\text {air }}$. Inside the human body, at temperature $310 \mathrm{~K}$, one can take $\varrho_{\text {air }}=1.11 \mathrm{~kg} \cdot \mathrm{m}^{-3}$. Let us also denote $\nu$ the air kinematic viscosity and $\mu=\varrho_{\text {air }} \nu$ the dynamic one. A standard value of $\mu$ is $10^{-5} \mathrm{~kg} \cdot \mathrm{m}^{-1} \cdot \mathrm{s}^{-1}$. The previous values can be found, for instance, in [1].

The spray itself is described by a probability density function (PDF), which we denote $f$. The PDF depends not only on $t$ and $x$, but also on the velocity $v \in \mathbb{R}^{2}$. In fact, $f$ can also depend on the particle radius $r$ (we here assume that the particles remain spherical) or the temperature. Let us emphasize that we do not take into account any phenomenon modifying the aerosol radius distribution (no collision, no abrasion, etc.). This means that the initial radius distribution is conserved. Therefore, the radius does not appear as a variable in the equations, but only as a parameter. The dependence of $f$ on $r$ allows to send particles with various radii in the computational domain $\Omega$. Dependence on temperature is not discussed here, but one can easily admit that, for instance, the temperature variation has a negligible influence on the phenomena in the airways, in standard conditions.

In the following, we assume that the aerosol is also an incompressible fluid very similar to water, so that its volume mass $\varrho_{\text {aero }}$ can be chosen as $\varrho_{\text {aero }}=1000 \mathrm{~kg} . \mathrm{m}^{-3}$. Then, for each particle with radius $r$, we can define its mass $m(r)=4 / 3 \pi r^{3} \varrho_{\text {aero }}$. One must keep in mind that, in the case of polluting sprays, the volume mass may be bigger, and the retroaction effect on the aerosol may then be significantly increased.

The quantity $f(t, x, v, r) \mathrm{d} r \mathrm{~d} v \mathrm{~d} x$ is then the number of particles at time $t$ inside the elementary volume centered at $(x, v, r)$ in the phase space. Eventually, here is the full system satisfied by $f, u$ and $p$ :

$$
\begin{aligned}
\partial_{t} u+\nabla_{x} \cdot(u \otimes u)-\nu \Delta_{x x} u+\frac{\nabla_{x} p}{\varrho_{\text {air }}} & =\frac{\mathcal{F}_{\text {aero }}}{\varrho_{\text {air }}}, \\
\nabla_{x} \cdot u & =0 \\
\partial_{t} f+\nabla_{x} \cdot(v f)+\nabla_{v} \cdot(a f) & =0
\end{aligned}
$$

where $a$ is the particle acceleration, mainly due here to the Stokes force exerted on the aerosol by the fluid, and is given by

$$
a(t, x, v, r)=\frac{6 \pi \mu r}{m(r)}(u(t, x)-v)
$$

and $\mathcal{F}_{\text {aero }}$ is the force exerted by the aerosol on the air, that is

$$
\mathcal{F}_{\text {aero }}(t, x)=-\iint_{\mathbb{R}^{2} \times \mathbb{R}_{+}} f(t, x, v, r) m(r) a(t, x, v, r) \mathrm{d} v \mathrm{~d} r=6 \pi \mu \iint_{\mathbb{R}^{2} \times \mathbb{R}_{+}} r f(t, x, v, r)(v-u(t, x)) \mathrm{d} v \mathrm{~d} r .
$$

The last term $\mathcal{F}_{\text {aero }}$ is the one we discuss in this paper. We shall often compare results when $\mathcal{F}_{\text {aero }}=0$ or is given by (5) to fill in (1). The system (1)-(5) was mathematically investigated (global in time existence) in [5] in a periodic in space framework, and without any dependence on $r$ (see also [2]).

We focus on a very simple geometry, namely a two-dimensional tube $\Omega=(0, L) \times(0, D)$, see Figure 1 . In fact, since the retroaction is first a local phenomenon (the particles first act on the fluid nearby), we do not need 


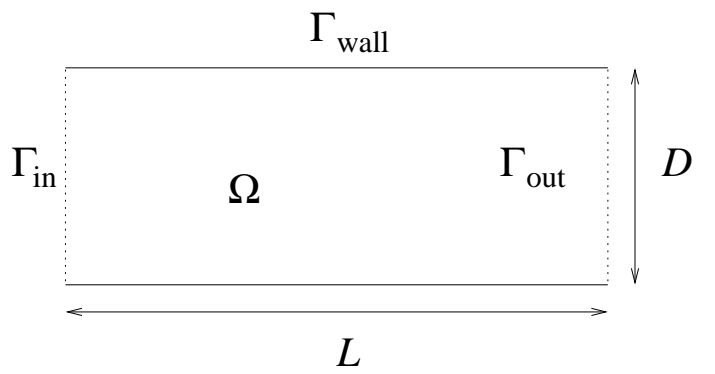

Figure 1. Standard tube with boundaries

to consider a biologically realistic computational domain. However, we will point out that some global major effects appear because of the retroaction. The boundary $\Gamma=\partial \Omega$ of the computational domain we consider is divided into three areas: the inlet $\Gamma_{\mathrm{in}}$, the outlet $\Gamma_{\text {out }}$ and the wall $\Gamma_{\text {wall }}$. We use $D=0.02 \mathrm{~m}$ and $L=0.5 \mathrm{~m}$. The value of $D$ we choose here has the typical order of magnitude of the diameter of a human trachea [21]. The choice of $L$ allows to capture the behaviours of the quantities we are interested in, away from the particles locations.

Equations (1)-(5) can then be supplemented with boundary conditions:

$$
\begin{aligned}
u(t, x) & =0 & & \text { if } x \in \Gamma_{\text {wall }}, \\
f(t, x, v, r) & =\tilde{f}(t, x, v, r) & & \text { if } x \in \Gamma_{\text {in }}, \\
f(t, x, v, r) & =0 & & \text { if } x \in \Gamma_{\text {wall }} \text { and } v \cdot n<0 .
\end{aligned}
$$

On the inlet and the outlet, we shall consider different boundary conditions on the fluid velocity, depending on the physical test cases we are interested in. These boundary conditions are explicitly given in each section. Condition (8) has been discussed in [6], $n$ being the outgoing normal vector to $\Gamma$ at the point of interest. In the airways, it means that whenever the particles hit the walls, they are immediately absorbed by the mucus.

\subsection{Numerical solving}

The airflow is solved by a standard $P^{2}$ (for the velocity) and $P^{1}$ (for the pressure) finite element computation (see [10] for instance), and the aerosol by a particle method (see [18] for example). We do not give any detail on the fluid computation, since (1)-(2) with standard boundary conditions are solved thanks to a Navier-Stokes routine using the Freefem++ software [14]. For the particle method, we have to distinguish the physical particles from the numerical ones. The total number of numerical particles $N$ is almost always much smaller than the number of real physical particles $N_{P}$. The PDF $f$ can discretized in the following way

$$
f(t, x, v, r)=\sum_{p=1}^{N} \omega_{p}(t) \delta_{x_{p}(t)}(x) \delta_{v_{p}(t)}(v) \delta_{r_{p}(t)}(r),
$$

where $t \mapsto\left(x_{p}(t), v_{p}(t), r_{p}(t)\right)$ is the trajectory of the numerical particle $p$ in the phase space, and $\omega_{p}(t)$ its representativity at time $t$. A numerical particle $p$ gives an average behaviour of a set of physical particles. The average value of $\omega_{p}$ is approximately of order $N_{P} / N$. Note that, when discretizing (1)-(3), the representativity $\omega_{p}$ only appears in the discretized version of (5), and not in the one of (4). We only use one constant value of $\omega_{p}$ in each numerical test.

In our numerical scheme, the coupling is explicit and solved only once at each time step. When there is no retroaction, in order to optimize the computational cost, one can set two different time steps, one for the fluid and one for the spray. Here, since we consider the spray retroaction on the air, we are forced to use exactly the same time step for the air and the particles to ensure that the momentum exchange between both 
phases happens whenever the velocities are modified. Note that, when we lower the time step, which satisfies a CFL-like condition, we obtain the same kind of numerical behaviours.

The mesh which was used in our computations has 1139 vertices and 1978 triangles, and $\delta x \simeq 5.10^{-3} \mathrm{~m}$, where $\delta x$ is a characteristic size of a cell.

In order to emphasize the retroaction effect, it is important to set a dynamic unbalance between the spray and the fluid. Indeed, if they both share the same velocity, the term $\mathcal{F}_{\text {aero }}$ is obviously equal to zero, and there is no retroaction at all. For instance, it is clear that the retroaction effect is strong in a situation when the particle and the fluid velocities are initially orthogonal. The retroaction then induces non-zero values on the initially nil velocity components for the fluid.

Nevertheless, even without precisely studying the latter, we recover most situations of interest on the retroaction by numerically investigating two cases. The first one, in Section 3, is dedicated to a spray which goes in an initially motionless fluid, and the second one, in Section 4 to a motionless spray which is put into a Poiseuille airflow. As the reader will see, most numerical results are not surprising, they only allow to control the model and code validity. But we also obtain a more surprising side effect: the wall deposition in the tube of the bigger particles $(r=50 \mu \mathrm{m})$. Such radii correspond to polluting particles, but also to some therapeutic aerosols.

\section{Moving SPRAY IN AN INITIALLY MOTIONLESS FLUID}

The first set of computational results is obtained in the case of one or several injections of particles in an initially motionless fluid. In each case, the particles are initially uniformly distributed on the tube inlet with an incoming horizontal velocity $v^{0}$. Initially, there is a distance of $5.10^{-3} \mathrm{~m}$ between the particles and the wall, since the retroaction effect induced by a particle very close the wall is almost nil: there, the air velocity remains equal to 0 , and the particles would immediately deposit.

\subsection{Choice of the representativity}

The PDF discretization induces two different numbers of particles: the number of numerical particles $N$ and the number of physical particles $N_{P}$. Those numbers are linked by the formula $N_{P}=N \omega_{p}$, assuming that the value of the representativity $\omega_{p}$ does not depend on the chosen numerical particle. The representativity allows to lead computations with $N$ numerical particles instead of $N_{P}$, which is of course less expensive.

We here take $v^{0}=1 \mathrm{~m} . \mathrm{s}^{-1}$ and $r=50 \mu \mathrm{m}$. On Figure 2, we plot the fluid velocity at coordinates $(0.01,0.01)$, for different values of $N$ and $\omega_{p}$, leading to the same number of physical particles $N_{P}=10^{10}$. This value of $N_{P}$ is the limit for most commercial nebulizers, see [17].

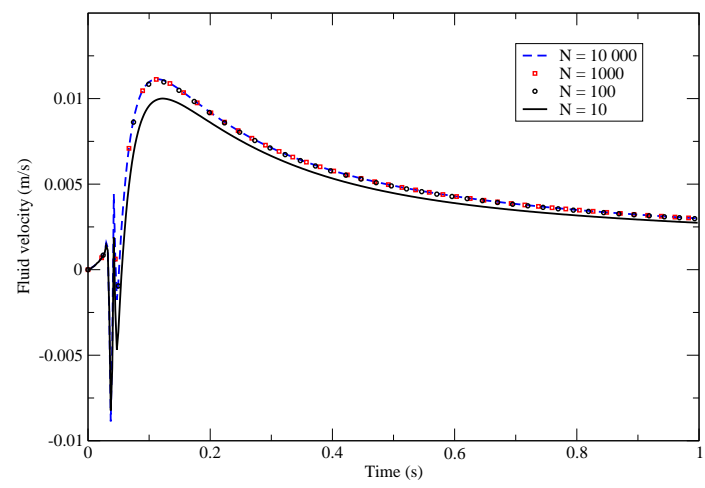

Figure 2. Cross-influence of $N$ and $\omega_{p}$ on the computation $\left(N_{P}=10^{10}\right)$ 
We observe that these different repartitions give the same results, as soon as there are enough numerical particles (e.g. $N \geq 100$ ). Therefore, in order to obtain faster simulations, we choose a small number of numerical particles $(N=100)$, and a big representativity $\omega_{p}=10^{8}$.

The next test aims to validate our numerical code, in the one injection case.

\subsection{Particle velocity without retroaction}

For these computations, we take $v^{0}=1 \mathrm{~m} \cdot \mathrm{s}^{-1}$ and $r=25 \mu \mathrm{m}$. When there is no retroaction, we can analytically compute the expression of each particle velocity $v_{p}$ with respect to $t$, as in [17]. In that case, it is clear that $v_{p}(t)=v^{0} \exp (-t / \tau)$, where

$$
\tau=\frac{m(r)}{6 \pi \mu r}=\frac{2 r^{2} \varrho_{\text {aero }}}{9 \mu} \simeq 1.3910^{-2} \mathrm{~s} .
$$

Then we plot the analytical particle velocity and the computed one on Figure 3, and check that the two curves are superimposed. Note that the curves do not depend on the chosen particle.

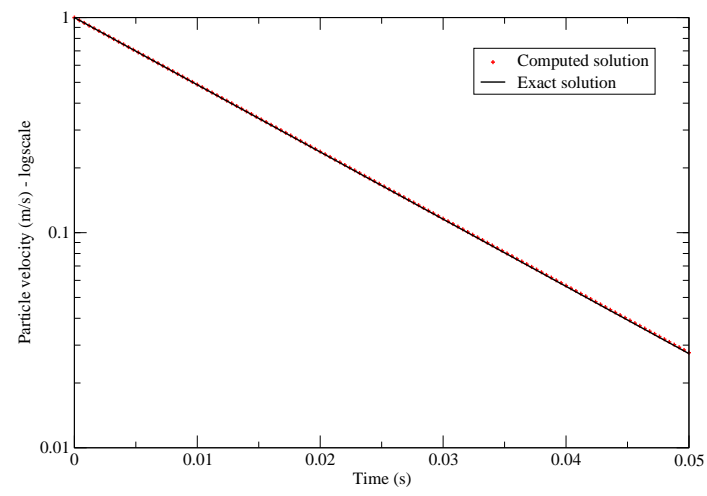

FiguRE 3. Analytical and computed particle velocity without spray retroaction

\subsection{One spray injection}

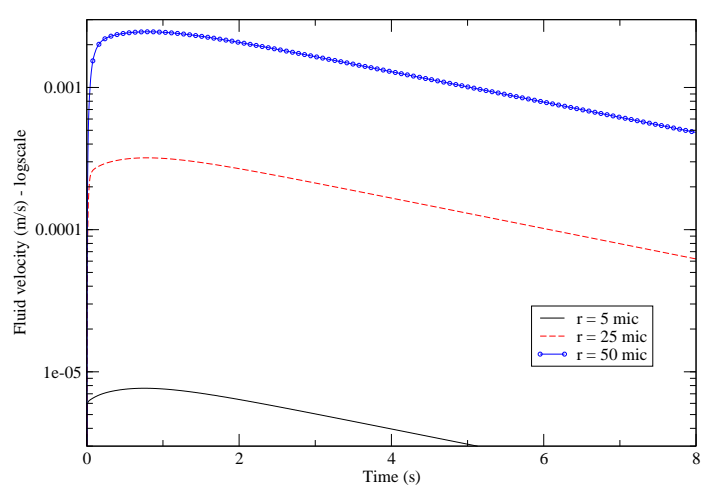

Figure 4. Air velocity in the tube center $\left(v^{0}=1 \mathrm{~m} \cdot \mathrm{s}^{-1}\right.$, different particle radii) 
We here study the effect of one injection, in the tube, of 100 numerical particles of various radii $(r=5,25$ or $50 \mu \mathrm{m}$ ), with representativity $\omega_{p}=10^{8}$, and initial velocity $v^{0}$ equal to 1 or $0.1 \mathrm{~m} . \mathrm{s}^{-1}$. As already stated, the particles are initially uniformly distributed at the tube inlet, but away from the wall. The set of particles and the wall are $5 \cdot 10^{-3} \mathrm{~m}$ apart.

\subsubsection{Particle radius influence}

We first plot, on Figure 4, the air velocity at $(0.206,0.01)$, i.e. on the tube axis, for different values of the radius. We recover that the bigger the particle is, the bigger its influence on the fluid is. Of course, this fact is intuitive, and we can begin to quantify it. When $r=50 \mu \mathrm{m}$, one can check that the fluid velocity can go up to $2.10^{-3} \mathrm{~m} . \mathrm{s}^{-1}$, whereas the fluid would be motionless when there is no spray retroaction.

\subsubsection{Injection velocity influence}

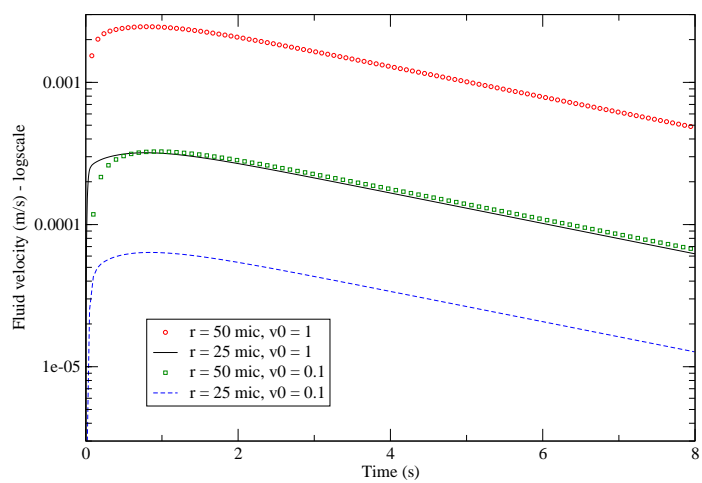

Figure 5. Air velocity in the tube center for two values of $v^{0}$ and different particles radii

We can also highlight the influence of the particles initial velocity $v^{0}$. Figure 5 shows the fluid velocity at the same point of the tube as in 3.3.1, for various radii and initial velocities. We recover the fact that particles injected with a bigger velocity have a greater influence on the fluid, and again that bigger particles also have a more significant retroaction on the fluid. This statement is again not surprising, and we can quantify it. When $r$ is fixed, the retroaction influence is systematically more significant when $v^{0}$ is bigger. It means that, when the injection spray velocities are high, the retroaction should be taken into account, or at least seriously considered.

\subsubsection{Fluid velocity field away from the spray}

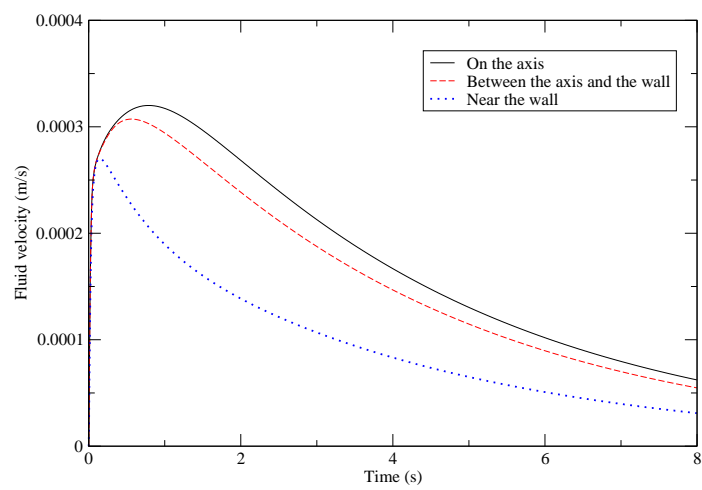

Figure 6. Air velocity at different locations in the tube $\left(v^{0}=1 \mathrm{~m} . \mathrm{s}^{-1}, r=25 \mu \mathrm{m}\right)$ 
In order to highlight the impact of the retroaction, we plot the fluid velocity at different locations of the tube, for particles of radius $r=25 \mu \mathrm{m}$ and velocity $v^{0}=1 \mathrm{~m} \cdot \mathrm{s}^{-1}$ on Figure 6 . In this figure, the coordinates of the chosen points are respectively $(0.390,0.167),(0.206,0.102)$ and $(0.107,0.007)$. We observe that the velocity is maximal on the axis of the tube, and it decreases when we get closer to the wall. This smaller effect of the retroaction near the wall is primarily due to the zero boundary condition on the fluid velocity on the wall.

Up to now, we focused on regions which particles do not cross before stopping in the fluid. Let us now emphasize the local effect, due to the presence of the particles, on the ambient fluid.

\subsubsection{Retroaction feedback on the spray}

When one takes the retroaction force $\mathcal{F}_{\text {aero }}$ into account in the Navier-Stokes equations, it induces a nonzero velocity in the fluid, which therefore impedes the particles to slow down as much as they would without retroaction. It is a second-order-like effect on the particles, since the spray drags the air, which then slows down less the particles. On Figure 7, we recover that the velocity of the particles (the one initially located at vertical coordinate 0.009 four our figure) decreases faster without the retroaction force. Nevertheless, the difference is at most $0.1 \%$ of the initial velocity.

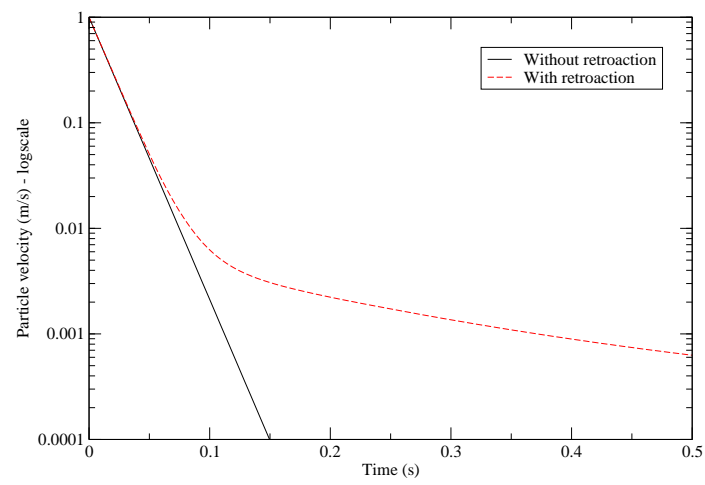

Figure 7. Particle velocity for $v^{0}=1 \mathrm{~m} \cdot \mathrm{s}^{-1}, r=25 \mu \mathrm{m}$, with and without retroaction

\subsubsection{Fluid velocity near the particles}

We here choose $v^{0}=1 \mathrm{~m} . \mathrm{s}^{-1}$ and $r=50 \mu \mathrm{m}$.
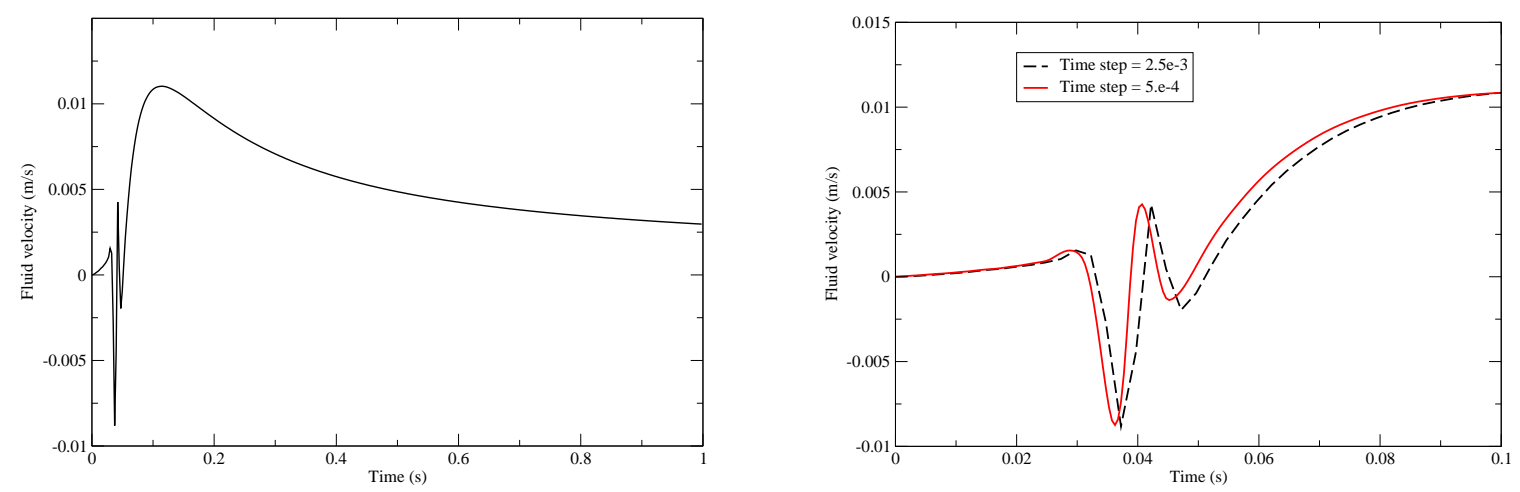

FIGURE 8. Fluid velocity near the particles: (a) global view and (b) zoom around $t=0.05 \mathrm{~s}$ 
Whereas the fluid velocity smoothly evolves far from the spray, the physical presence of the particles strongly disturbs the airflow. If we plot the fluid velocity at the point of coordinates $(0.0276,0.0133)$, we can observe the influence of the particles passing at this point. This is shown on Figure 8a.

More precisely, we observe a jump on the fluid velocity, which can be explained [15] by the incompressibility of the air and the fact that the particles are hard spheres. Indeed, when a particle goes across a cell, it takes up a small amount equivalent to its volume, which cannot be occupied by the fluid. When the particle arrives, the fluid is rejected towards the inlet because of the viscosity, to ensure the volume conservation. Then the particle begins to drag the downstream fluid.

We note on Figure $8 \mathrm{~b}$ that the computation near the jump, at time 0.05 , is stable with respect to the time step. We also emphasize that the model prevents the choice of a finer mesh. Indeed, in that case, a particle incoming in a cell would fill more than $10 \%$ of the cell volume, which is not allowed by the standard thin spray assumption for respiratory aerosols (the volume fractions are not involved in the equations).

\subsubsection{Fluid velocity field}

From a more global point of view, the previous local effect also generates a lateral fluid recirculation near the walls (see Figure 9). Since the spray is distributed along the section of the tube with a lateral gap near the walls, it pushes the fluid in the central part, whereas the fluid escapes (with negative velocities) near the walls.

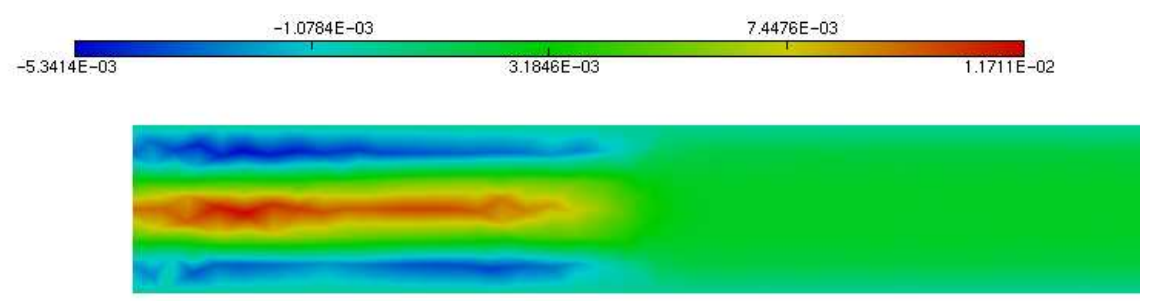

FIGURE 9. Fluid velocity in the whole domain

\subsection{Ten spray injections}

The computations are made with the same number of physical and numerical particles as in 3.3. Hence, the representativity of each numerical particle is $\omega_{p}=10^{7}$, and there are 10 successive injections of 100 numerical particles, to recover the same number of physical particles. Each injection happens at every ten time steps. This framework seems more realistic from a medical point of view: there is no massive spray inhalation at exactly the same time, it may last for some time. We pick $v^{0}=1 \mathrm{~m} \cdot \mathrm{s}^{-1}$.

\subsubsection{Fluid velocities}

On Figure 10, we plot the fluid velocity in the one-injection and the ten-injection cases, at the same point near the tube inlet $(0.0276,0.0133)$, i.e. which the particles go nearby. We observe that, although the particle number is the same and the injections happen quasi-simultaneously, the velocity jump occurring in the one-injection case because of the injection tends to disappear with the number of injections, while keeping $N_{P}$ constant. Nevertheless, the maximal value reached by the fluid velocity is of the same order, and, at the beginning of the computation, it is relevant to keep the retroaction force $\mathcal{F}_{\text {aero }}$ in the model.

\subsubsection{Particle velocities}

Meanwhile, if we compare the velocity of a particle in the one-injection case and of a particle in the first (out of ten) injection, initially located at the same point near the tube inlet (same coordinates as in 3.3.4), we recover that the two curves are superimposed (Figure 11a). This may be surprising since, at some time, the downstream fluid should see the effect of the other waves of particles. That only means that the retroaction feedback on the particle observed in 3.3.4 can probably be neglected. 


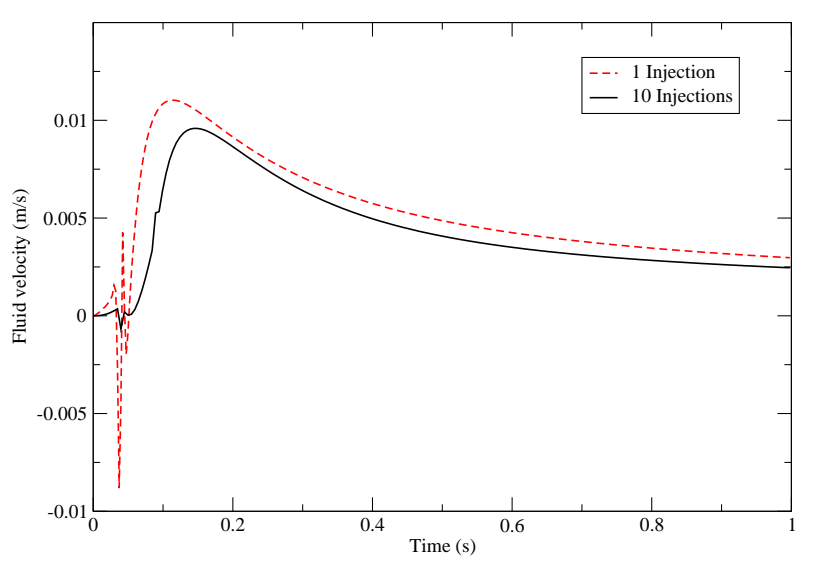

Figure 10. Fluid velocity: one injection vs. ten injections
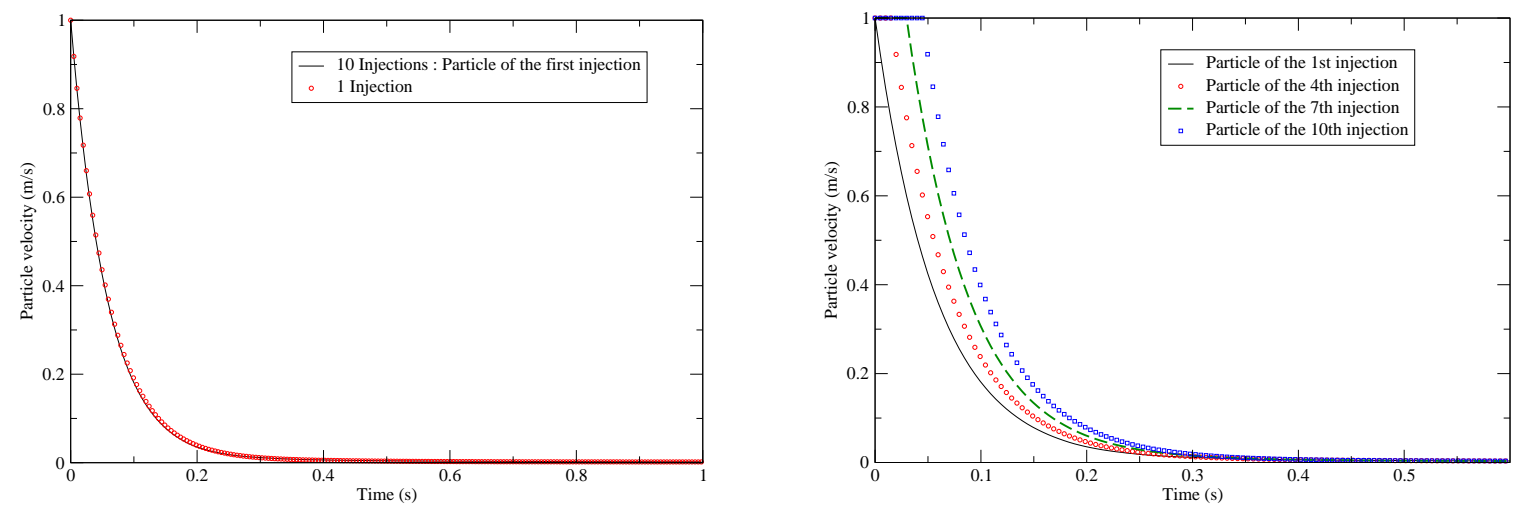

FiguRe 11. Particle velocities: (a) one injection vs. ten injections (b) various injections (out of 10)

Moreover, we can plot the velocities of particles initially located at the same point but belonging to different injections (Figure 11b). In this case, there is no significant difference (except for the time-lag) between particles of the first injection or of the last. This complies with our conclusion about neglecting the effect of the retroaction feedback on the particles.

\section{Initially motionless particles in a Poiseuille Airflow}

Once again, we use the same tube and mesh as in Section 2.2. This time, we consider a Poiseuille airflow and 100 initially motionless numerical particles with representativity $\omega_{p}=10^{8}$. Those are uniformly distributed along a vertical section of the tube, at a distance $\ell=0.01 \mathrm{~m}$ from the inlet $\Gamma_{\text {in }}$.

It is well-known that, for any $u^{0}>0$, which then appears as the maximal (horizontal) air velocity on $\Gamma_{\text {in }}$, the function $(u, p)$ defined by

$$
u\left(t, x_{1}, x_{2}\right)=\left(u^{0}\left[1-\left(\frac{x_{2}-D / 2}{D / 2}\right)^{2}\right], 0\right), \quad p(t, x, y)=\left(-2 x_{1}+L\right) \frac{4 \nu u^{0}}{D^{2}},
$$



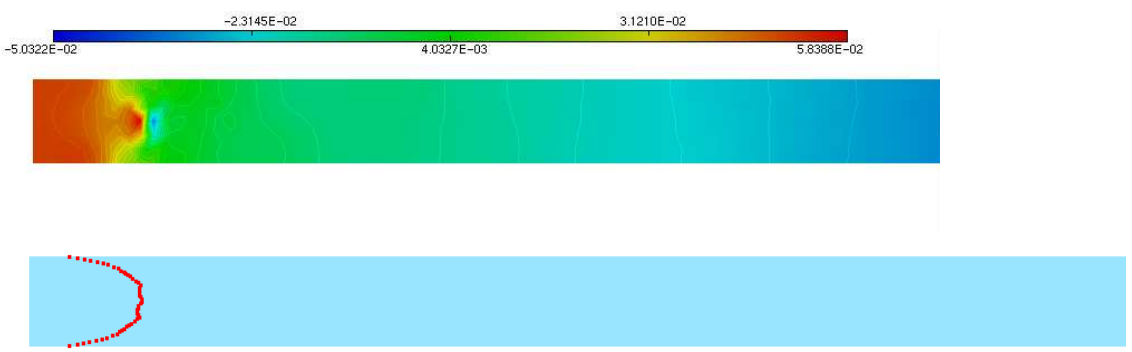

Figure 12. (a) Fluid pressure and (b) particles position, at $t \simeq 0.5 \mathrm{~s}$

solves the Navier-Stokes equations (1)-(2) with $\mathcal{F}_{\text {aero }}=0$, and the boundary conditions $(6)$ and

$$
p=\frac{4 \nu u^{0} L}{D^{2}} \quad \text { on } \Gamma_{\text {in }}, \quad p=-\frac{4 \nu u^{0} L}{D^{2}} \quad \text { on } \Gamma_{\text {out }} .
$$

There is no term $\partial u / \partial n$ in the previous boundary conditions, because this quantity is nil on both $\Gamma_{\text {in }}$ and $\Gamma_{\text {out }}$. When this solution is disturbed, the pressure boundary conditions are modified by taking into account the quantity $\nu \partial u / \partial n-p n$ instead of $p$ only.

The fluid is set with the initial condition on $u$ and $p$ given by (9). The spray induces a perturbation of the fluid Poiseuille profile.
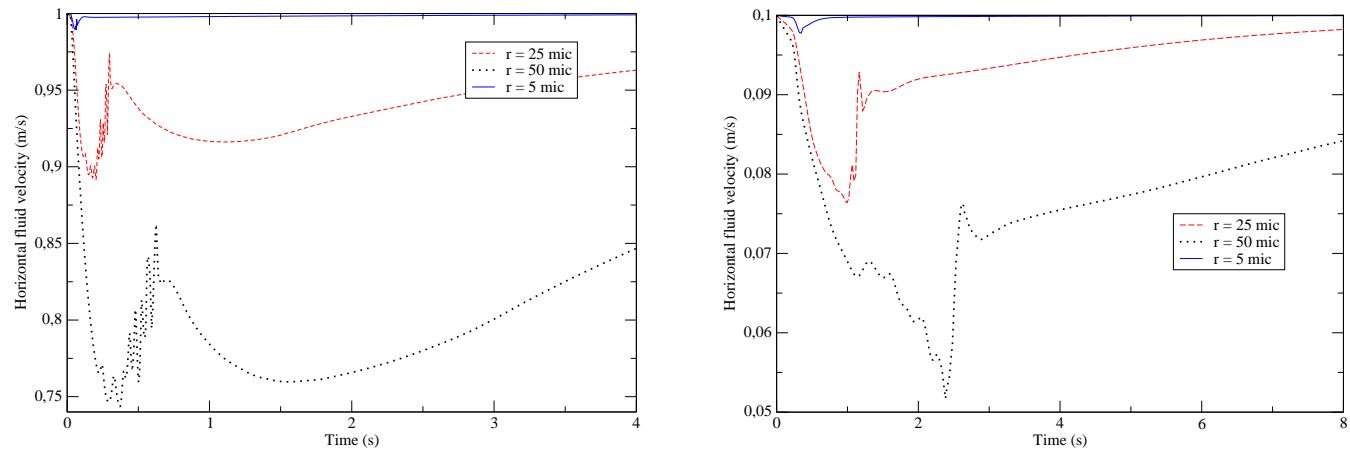

FIgURE 13. Horizontal fluid velocity for (a) $u^{0}=1$, (b) $u^{0}=0.1 \mathrm{~m} \cdot \mathrm{s}^{-1}$

\subsection{Fluid pressure and velocity field}

Let us choose $u^{0}=1 \mathrm{~m} \cdot \mathrm{s}^{-1}$ and $r=50 \mu \mathrm{m}$. Figure 12 presents the fluid pressure and the position of the particles at the same time $t \simeq 0.5 \mathrm{~s}$. If there is no retroaction, the pressure isolines permanently remain vertical. With the retroaction, the pressure isolines on Figure 12a are clearly not vertical near the particles, they are influenced by the spray wave shown on Figure 12b.

In order to quantify the effect of the retroaction, we take different values in the following tests for the maximal air velocity: $u^{0}=1 \mathrm{~m} . \mathrm{s}^{-1}$ and $u^{0}=0.1 \mathrm{~m} . \mathrm{s}^{-1}$, and various particle radii: $r=5 \mu \mathrm{m}, r=25 \mu \mathrm{m}$ and $r=50 \mu \mathrm{m}$ and plot the fluid velocity at $(0.0276,0.0133)$, near the inlet.

The spray clearly has an effect on the fluid, as one can see on Figures 13 and 14, for the bigger particles. Note that the vertical velocity component goes back to zero, but, for bigger particles, the time during which it is quite different from 0 is not negligible. 

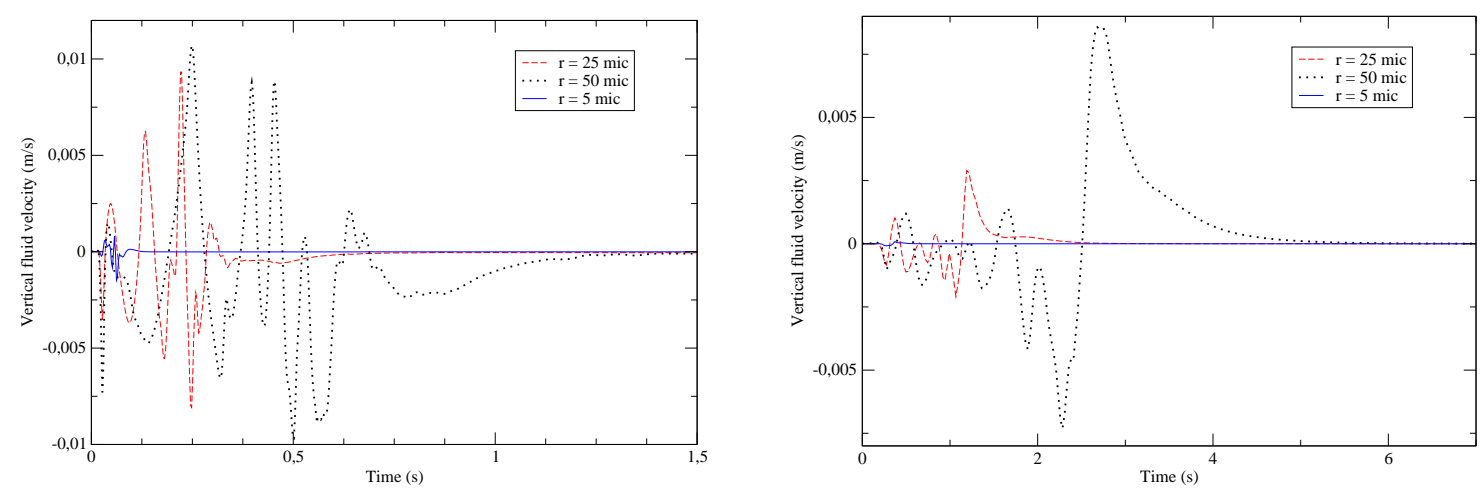

Figure 14. Vertical fluid velocity for (a) $u^{0}=1$, (b) $u^{0}=0.1 \mathrm{~m} \cdot \mathrm{s}^{-1}$

\subsection{Particle velocity}

We focus on the particle which is initially located on the tube axis $y=D / 2$. As in Section 3 , we first study the particle velocity in the main flow direction, i.e. the horizontal component of the particle velocity, for various radii of the particle. Its behaviour is given on Figure 15a for $u^{0}=1 \mathrm{~m} \cdot \mathrm{s}^{-1}$ and on Figure 15b for $u^{0}=0.1 \mathrm{~m} . \mathrm{s}^{-1}$.
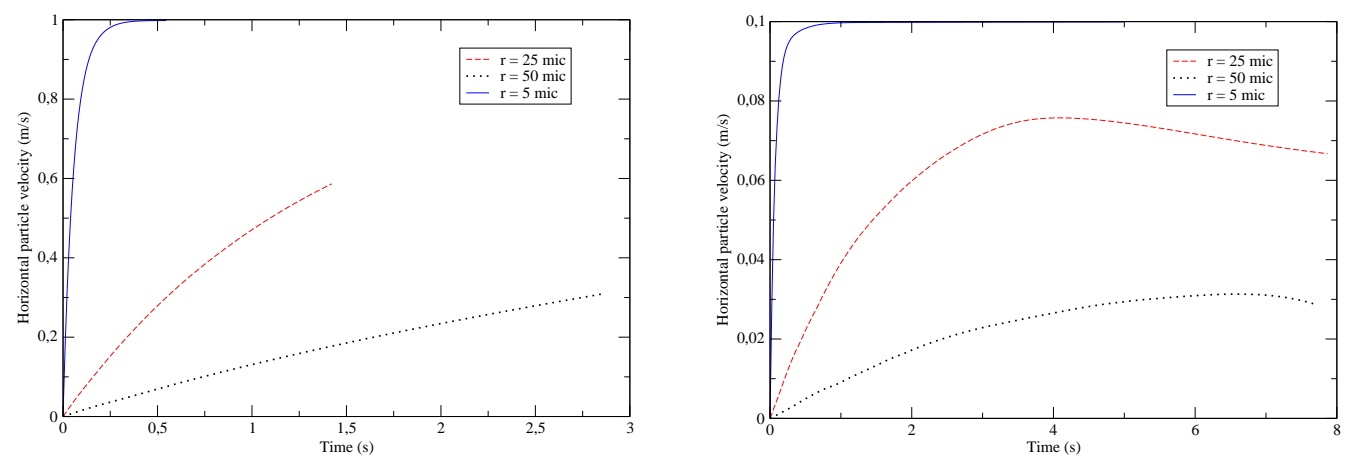

Figure 15. Horizontal particle velocity for (a) $u^{0}=1$, (b) $u^{0}=0.1 \mathrm{~m} . \mathrm{s}^{-1}$

The curves stop before the final computational time, because the particle of interest somehow left the domain $\Omega$ before its velocity reaches an equilibrium. Obviously, the velocity of the lighter particle almost immediately reaches $u^{0}$, but this is not the case for the two other ones. If the situation for $u^{0}=1 \mathrm{~m} \cdot \mathrm{s}^{-1}$ is inconclusive, it is clear that, for $u^{0}=0.1 \mathrm{~m} . \mathrm{s}^{-1}$, the asymptotic velocities are not $u^{0}$. That suggests that the particle is not on the tube axis anymore, i.e. the particle velocity has a non-zero vertical component, which is consistent with the non-zero vertical fluid velocity. We check this fact on Figure $16 \mathrm{a}$ for $u^{0}=1 \mathrm{~m} \cdot \mathrm{s}^{-1}$ and on Figure $16 \mathrm{~b}$ for $u^{0}=0.1 \mathrm{~m} \cdot \mathrm{s}^{-1}$.

When $u^{0}=1 \mathrm{~m} \cdot \mathrm{s}^{-1}$, the particle goes out from $\Omega$ whatever the radius is, but the retroaction should be taken into account, mainly because of the vertical component of the velocity. On the other hand, when $u^{0}=0.1 \mathrm{~m} . \mathrm{s}^{-1}$, one can see that the big particle and the intermediate one leave the domain $\Omega$ at approximately the same time. That implies that the big one has deposited. That would never have happened without retroaction: no particle should deposit in that case. We check that there is some spray deposition on Figure 17. 

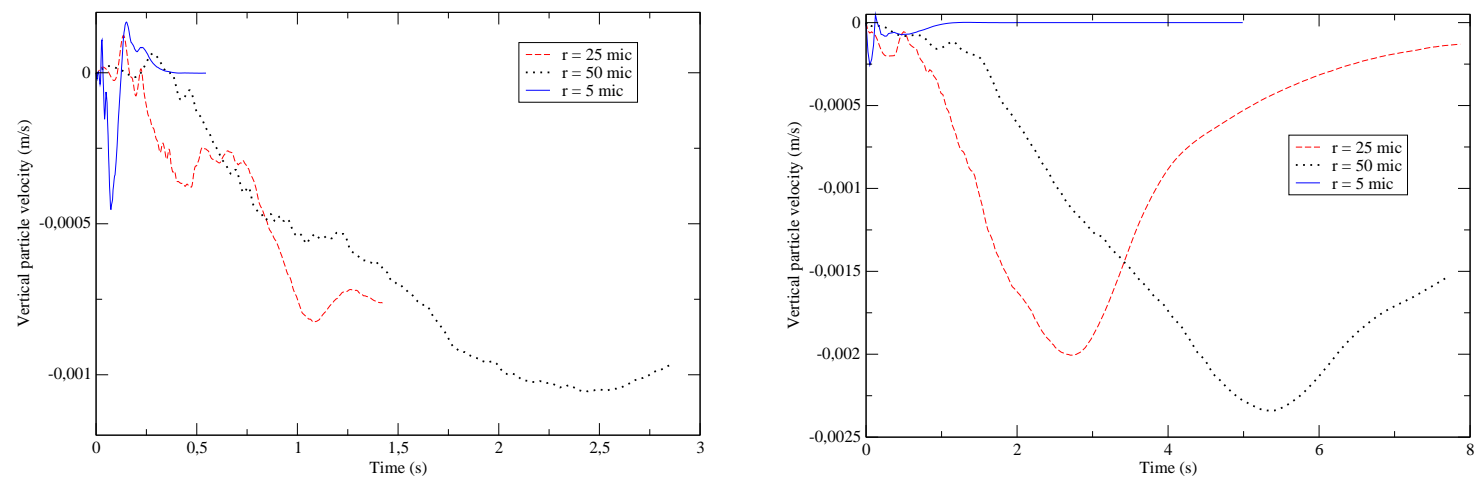

FiguRE 16. Vertical particle velocity for (a) $u^{0}=1$, (b) $u^{0}=0.1 \mathrm{~m} \cdot \mathrm{s}^{-1}$

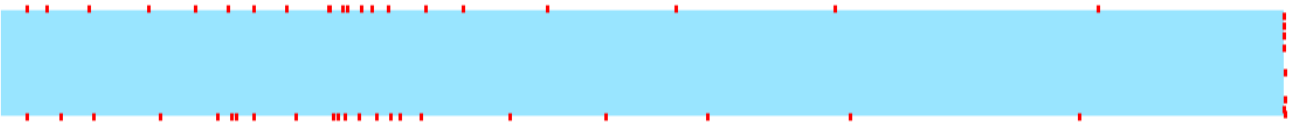

Figure 17. Particle deposition at final time for $u^{0}=1 \mathrm{~m} \cdot \mathrm{s}^{-1}$ and $r=50 \mu \mathrm{m}$

\section{Conclusion}

In this work, we investigated the influence of the retroaction force $\mathcal{F}_{\text {aero }}$ in the Navier-Stokes equations. This term ensures the conservation of the total momentum of the spray-fluid model. The spray may interfere with the fluid in many situations.

For the numerics, we used some biologically relevant values for the velocities and radii. It is quite clear that, for radii smaller than $25 \mu \mathrm{m}$, the retroaction is negligible, whatever the velocities are. On the contrary, our computations showed that, for radii bigger than $25 \mu \mathrm{m}$, in most of the situations, the retroaction should not be neglected to properly and accurately capture the fluid behaviour, mainly if one of the velocities is high. Indeed, it clearly has an influence on the deposition. Of course, there is also an influence of the aerosol concentration, but it is not studied here: we only focused on the most concentrated types of aerosols, corresponding to $10^{10}$ physical particles.

With this deposition effect, the retroaction seems to favour the filter role that the upper airways have to play on the bigger particles. This fact has clearly to be confirmed on more realistic three-dimensional computations, for example, in a branched structure, but especially when one takes into account the physical phenomena known as responsible for the deposition process: the gravitational sedimentation and the diffusive displacement, see [8].

Acknowledgement. The authors want to thank Anne Devys for making the working atmosphere productive and lovely. They are also grateful to Sébastien Martin and Bertrand Maury for the very helpful scientific discussions which took place during Cemracs, and Frédéric Hecht and Mourad Ismail for their support for the Freefem++ use for the project.

\section{REFERENCES}

[1] J.E. Agnew, D. Pavia, and S.W. Clarke. Aerosol particle impaction in the conducting airways. Phys. Med. Biol., 29:767-777, 1984.

[2] O. Anoshchenko and A. Boutet de Monvel-Berthier. The existence of the global generalized solution of the system of equations describing suspension motion. Math. Methods Appl. Sci., 20(6):495-519, 1997. 
[3] L. Baffico, C. Grandmont, and B. Maury. Multiscale modeling of the respiratory tract. Math. Models Methods Appl. Sci., 20(1):59-93, 2010.

[4] C. Baranger, L. Boudin, P.-E. Jabin, and S. Mancini. A modeling of biospray for the upper airways. ESAIM Proc., 14:41-47, 2005.

[5] L. Boudin, L. Desvillettes, C. Grandmont, and A. Moussa. Global existence of solutions for the coupled Vlasov and NavierStokes equations. Differential Integral Equations, 11-12:1247-1271, 2009.

[6] L. Boudin and L. Weynans. Spray impingement on a wall in the context of the upper airways. ESAIM Proc., 23:1-9, 2008.

[7] J. K. Comer, C. Kleinstreuer, and Z. Zhang. Flow structures and particle deposition patterns in double bifurcation airway models. Part 2. Aerosol transport and deposition. J. Fluid Mech., 435:55-80, 2001.

[8] P. Gehr and J. Heyder, editors. Particle-lung interactions. Lung biology in health and disease. Marcel Dekker, Inc., 2000.

[9] T. Gemci, T. E. Corcoran, and N. Chigier. A numerical and experimental study of spray dynamics in a simple throat model. Aerosol Sci. Technol., 36:18-38, 2002.

[10] V. Girault and P.-A. Raviart. Finite element methods for Navier-Stokes equations, volume 5 of Springer Series in Computational Mathematics. Springer-Verlag, Berlin, 1986. Theory and algorithms.

[11] C. Grandmont, Y. Maday, and B. Maury. A multiscale/multimodel approach of the respiration tree. In New trends in continuum mechanics, volume 3 of Theta Ser. Adv. Math., pages 147-157. Theta, Bucharest, 2005.

[12] C. Grandmont, B. Maury, and A. Soualah. Multiscale modelling of the respiratory track: a theoretical framework. ESAIM Proc., 23:10-29, 2008.

[13] J.B. Grotberg. Respiratory fluid mechanics and transport processes. Annu. Rev. Biomed. Eng., 3:421-457, 2001.

[14] F. Hecht, A. Le Hyaric, K. Ohtsuka, and O. Pironneau. Freefem++, finite elements software, http://www.freefem.org/ff++/.

[15] F. Lagoutière, N. Seguin, and T. Takahashi. A simple 1D model of inviscid fluid-solid interaction. J. Differential Equations, 245(11):3503-3544, 2008.

[16] B. Mauroy. Hydrodynamics in the lungs, relations between flows and geometries. PhD thesis, ENS Cachan, 2004.

[17] A. Moussa. Aerosols in the human lung. PhD thesis, ENS Cachan, 2009.

[18] E. Sonnendrücker, J. Roche, P. Bertrand, and A. Ghizzo. The semi-Lagrangian method for the numerical resolution of the Vlasov equation. J. Comput. Phys., 149(2):201-220, 1999.

[19] A. Soualah-Alilah. Modélisation mathématique et numérique du poumon humain. PhD thesis, Université Paris-Sud 11, 2007.

[20] C. Vannier. Modélisation mathématique du poumon humain. PhD thesis, Université Paris-Sud 11, 2008.

[21] E.R. Weibel. The pathway for oxygen: structure and function in the mammalian respiratory system. Harvard University Press, 1984. 